\title{
Associação entre excesso de peso, obesidade, dor músculo- -esquelética e osteoartrose em cuidados de saúde primários: estudo transversal
}

Andreia Baptista Marques*

\section{RESUMO}

Objetivos: Avaliar a associação de excesso de peso, obesidade, dor músculo-esquelética e osteoartrose em cuidados de saúde primários.

Tipo de estudo: Observacional, analítico, transversal.

Local: Unidades de saúde dos Agrupamentos de Centros de Saúde da Administração Regional de Saúde de Lisboa e Vale do Tejo. População: Indivíduos com $\geq 21$ anos de idade que, em 2014, consultaram um médico de família.

Métodos: Os dados para a análise foram recolhidos do Sistema de Informação da Administração Regional de Saúde. Os códigos L13, L15, L89, L90, T82 e T83 do ICPC-2 foram descritos por género e grupo etário. As medidas descritivas e o teste de qui-quadrado foram aplicados para avaliar diferenças de prevalência entre variáveis, sendo considerado um nível de significância de $5 \%$. Resultados: A prevalência nos 1.742 .470 participantes no estudo de excesso de peso e de obesidade foi de 7,42\% e $8,69 \%$. A prevalência de sintoma/queixa da anca foi de $1,51 \%$, de sintoma/queixa do joelho $3,25 \%$, de coxartrose $2,57 \%$ e de gonartrose $5,46 \%$. Foi encontrada uma associação positiva entre o excesso de peso e sintoma/queixa da anca (OR=1,07; IC95\%: 1,02$1,12, p<0,0001)$, sintoma/queixa do joelho (OR=1,15; IC95\%: 1,12-1,19, $p<0,0001)$, coxartrose $(O R=2,17 ; \mathrm{IC} 95 \%: 2,11-2,24$, $p<0,0001)$ e gonartrose (OR=2,18; IC95\%:2,14-2,23, $p<0,0001)$ e entre a obesidade e sintoma/queixa da anca (OR=1,23; IC95\%: $1,18-1,28, p<0,0001)$, sintoma/queixa do joelho (OR=1,62; IC95\%: 1,58-1,66, $p<0,0001)$, coxartrose (OR=2,57; IC95\%: $2,50-$ $2,63, p<0,0001)$ e gonartrose (OR=3,69; IC95\%: 3,63-3,75, $p<0,0001)$.

Conclusões: A associação positiva entre o excesso de peso e a obesidade e sintoma/queixa da anca, sintoma/queixa do joelho, osteoartrose da anca e osteoartrose do joelho e confirmam para a população portuguesa os resultados de estudos internacionais.

Palavras-chave: Obesidade; Dor músculo-esquelética; Osteoartrose; Associação; Cuidados de saúde primários.

\section{INTRODUÇÃO}

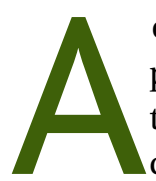
obesidade, a dor e as doenças reumáticas são problemas de saúde muito frequentes e potencialmente causadores de perda da qualidade de vida e de incapacidade, além de provocarem elevados custos sociais e económicos, motivos pelos quais têm sido alvo da atenção dos cidadãos, dos profissionais e dos administradores de saúde e também dos decisores políticos.

*Interna do Ano Comum no Hospital de Reynaldo dos Santos, Vila Franca de Xira
Em Portugal, a Direção-Geral da Saúde implementou desde 2001 diversas intervenções no âmbito destes problemas de saúde, como foram o Plano Nacional de Luta Contra a Dor, o Programa Nacional de Combate à Obesidade e o Programa Nacional Contra as Doenças Reumáticas e, atualmente, no âmbito do Programa Nacional de Saúde, os programas prioritários nas áreas da Promoção da Alimentação Saudável e da Promoção da Atividade Física.

A obesidade, definida pela Organização Mundial da Saúde (OMS) como uma acumulação anormal ou ex- 
cessiva de gordura corporal, que pode atingir graus capazes de afetar a saúde, é uma epidemia global bem reconhecida, com uma tendência de crescimento preocupante. Segundo as suas estimativas de 2014, mais de 1,9 mil milhões de adultos em todo o mundo tinham excesso ponderal (39\% da população mundial adulta $38 \%$ dos homens e $40 \%$ das mulheres) e, destes, mais de 600 milhões eram obesos (13\% da população mundial adulta $-11 \%$ dos homens e $15 \%$ das mulheres). A nível mundial, a prevalência da obesidade mais do que duplicou entre 1980 e 2014. ${ }^{1}$

A obesidade, principalmente se acompanhada de gordura visceral, está associada a uma inflamação crónica sistémica de baixo grau e está associada a um risco elevado para um grande número de doenças crónicas, nas quais se incluem tanto condições inflamatórias como degenerativas do sistema músculo-esquelético, como é especialmente o caso da osteoartrose, ${ }^{1-2}$ doença degenerativa da cartilagem e do osso subcondral caracterizada por estreitamento da entrelinha articular, devido à degradação enzimática da matriz, osteofitose e esclerose óssea subcondral. É uma doença de etiologia multifatorial, cujas manifestações clínicas são a dor e a disfunção articular, podendo levar à incapacidade e que afeta mais pessoas do que qualquer outra doença articular, aumentando a sua prevalência com a idade. ${ }^{2}$

A relação entre a obesidade e a osteoartrose, principalmente a do joelho, está bem demonstrada em vários estudos, tanto transversais como longitudinais, alguns deles com recurso a meios complementares de diagnóstico, como a ressonância magnética nuclear. Relativamente a outras articulações, como as da coluna lombar, das ancas, dos tornozelos e dos pés, é menor o número de estudos relacionando a obesidade e a osteoartrose e os seus resultados revelam existir uma menor associação entre elas..$^{2-3} \mathrm{~A}$ obesidade, ao provocar rotura mecânica, incluindo a compressão da articulação e alterações do alinhamento, pode desencadear a osteoartrose. Contudo, esta associação entre a obesidade e a osteoartrose vai para além da sobrecarga mecânica causada pelo excesso de peso, o que é evidente no caso da osteoartrose das mãos, cujas articulações não estão sujeitas a sobrecarga e, apesar disso, são geralmente afetadas pela doença, sugerindo ser o estado inflamatório crónico presente na obesidade um dos seus fatores etiológicos. ${ }^{2}$
A dor relacionada com a obesidade é também um problema de saúde cada vez mais comum. Só nos Estados Unidos da América existem 100 milhões de pessoas que têm dor crónica e 150 milhões que têm excesso de peso ou obesidade. No caso específico da dor músculo-esquelética relacionada com a obesidade, e da mesma maneira que foi constatado relativamente à osteoartrose, a associação entre elas vai para além da sobrecarga mecânica, estando provavelmente também relacionada com a inflamação crónica. ${ }^{4}$

O objetivo deste estudo foi a avaliação da associação do excesso de peso, da obesidade, da dor músculo-esquelética e da osteoartrose (anca e joelho) em utilizadores com $\geq 21$ anos de idade dos cuidados de saúde primários (CSP) da Administração Regional de Saúde de Lisboa e Vale do Tejo (ARSLVT).

\section{MÉTODOS}

\section{Tipo de estudo}

Esta investigação seguiu um desenho de estudo observacional analítico transversal.

\section{Participantes}

Os participantes foram os indivíduos com $\geq 21$ anos de idade utilizadores das consultas de medicina geral e familiar (MGF), inscritos nas unidades de saúde dos 15 Agrupamentos de Centros de Saúde (ACeS) da Administração Regional de Saúde de Lisboa e Vale do Tejo (ARSLVT) que, durante o ano de 2014, tiveram pelo menos uma consulta efetivada e cuja informação sociodemográfica e clínica está registada na base de dados do Sistema de Informação da Administração Regional de Saúde (SIARS). O carregamento dos dados sociodemográficos é feito administrativamente e o dos dados clínicos é feito pelos médicos de MGF durante as consultas. Um problema ativo é aquele que é diagnosticado e codificado segundo a Classificação Internacional de Cuidados Primários-Segunda Edição (ICPC-2), ${ }^{5}$ sendo considerado ativo enquanto estiver presente. Os problemas ativos são registados nos diversos programas informáticos de apoio à prática clínica em utilização nas unidades de saúde, os quais estão conectados ao SIARS, onde toda a informação fica armazenada em base de dados.

\section{Aspetos éticos}

O projeto do estudo foi apresentado à Comissão de 
Ética para a Saúde e ao Presidente do Conselho Diretivo da ARSLVT, solicitando a aprovação e a autorização para a sua realização. Após a sua obtenção foram solicitados os dados necessários para a sua realização ao Núcleo de Informática - Sistemas de Informação, da ARSLVT.

\section{Recolha de dados}

Os dados para a análise foram os recolhidos da base de dados do SIARS a partir dos indivíduos com $\geq 21$ anos de idade, utilizadores das consultas de MGF durante o ano de 2014 (com um registo SOAP - Subjetivo, Objetivo, Avaliação e Plano, associado a um contacto direto) e que durante esse mesmo período apresentaram com estado ativo, na lista de problemas (códigos das rubricas da ICPC- $2^{5}$ que estiverem escritos no "A" do registo SOAP), os seguintes problemas de saúde:

- Relacionados com um IMC igual ou superior a 25:

- T82 - Obesidade (IMC igual ou superior a 30)

- T83 - Excesso de peso (IMC superior a 25 mas inferior a 30)

- Relacionados com a dor músculo-esquelética:

- L13 - Sintoma/Queixa da anca

- L15 - Sintoma/Queixa do joelho

- Relacionadas com a osteoartrose:

- L89 - Osteoartrose da anca

- L90 - Osteoartrose do joelho

A recolha de dados foi feita por género e grupo etário (de 10 em 10 anos), dado que a informação do SIARS apenas foi disponibilizada de forma agregada.

Para cada um dos códigos relacionados com a dor músculo-esquelética (L13, L15) e a osteoartrose (L89, L90) a recolha foi feita respetivamente para a totalidade dos utilizadores, para os utilizadores com excesso de peso (T83) e para os utilizadores com obesidade (T82).

\section{Análise estatística}

Na análise descritiva dos dados, estes são apresentados sob a forma de tabelas de frequências absolutas e relativas. Foram aplicados testes de qui-quadrado de Pearson para avaliar as diferenças de prevalência entre as variáveis, sendo o resultado dos testes apresentado como odds ratio (OR) com intervalos de confiança a 95\% (IC95\%), considerando um nível de significância de 0,05 ( $\mathrm{p} \leq 0,05)$. A análise estatística foi realizada através do programa estatístico GraphPad Prism $5 \circledR$.

\section{RESULTADOS}

Dos 3.774.057 inscritos na ARSLVT, 57,8\% ( $n=2.182 .557)$ frequentaram a consulta de MGF durante o ano de 2014. A idade de 43.058 destes utilizadores foi indeterminada e 397.029 tinham menos de 21 anos de idade, pelo que a análise foi efetuada a partir dos dados dos restantes $1.742 .470-39,86 \%(n=694.612)$ do género masculino $(\mathrm{H})$ e $60,14 \%(n=1.047 .858)$ do género feminino (M).

Destes 1.742 .470 utilizadores, $1.461 .711[83,89 \%$ $(\mathrm{H}=83,31 \%, \mathrm{M}=84,27 \%)]$ apresentavam um IMC menor que 25 (IMC<25) (sem excesso ponderal - grupo de referência), enquanto 129.378 [7,42\% $(\mathrm{H}=8,63 \%$, $\mathrm{M}=6,33 \%$ )] apresentavam um IMC entre 25 e 29,9 (IMC=25-29,9) (excesso de peso-T83) e 151.381 [8,69\% $(\mathrm{H}=8,06 \%, \mathrm{M}=9,10 \%)]$ apresentavam um IMC igual ou maior que 30 (IMC $\geq 30$ ) (obesidade - T82) (Quadro I).

\section{Sintoma/queixa da anca (L13)}

Apresentaram sintoma/queixa da anca $1,51 \%$ dos utilizadores ( $n=26.318$ ), dos quais $1,04 \%$ do género masculino ( $n=7.228)$ e $1,82 \%$ do género feminino $(n=19.090)$. A prevalência de L13 nos utilizadores com IMC $<25$ foi de $1,47 \%$ ( $\mathrm{H}=1,04 \%, M=1,76 \%)$, nos com IMC=25-29,9 foi de $1,58 \%(\mathrm{H}=1,01 \%, \mathrm{M}=2,06 \%)$ e naqueles com IMC $\geq 30$ foi de $1,80 \%$ ( $\mathrm{H}=1,07 \%, \mathrm{M}=2,23 \%)$ (Quadro II).

Relativamente a L13, a diferença entre a prevalência dos utilizadores com IMC $<25$ e com IMC=25-29,9 é estatisticamente significativa (OR=1,07; IC95\%: 1,02-1,12; $p<0,0001)$, assim como também o é a dos com IMC $<25$ e com IMC $\geq 30$ (OR=1,23; IC95\%: 1,18-1,28; $p<0,0001)$. De referir que, no género masculino, esta diferença não é estatisticamente significativa (Quadro III).

\section{Sintoma/queixa do joelho (L15)}

Apresentaram sintoma/queixa do joelho 3,25\% ( $n=56.703)$ dos utilizadores, dos quais $2,88 \%(n=20.030)$ do género masculino e $3,50 \%$ ( $n=36.673)$ do género feminino. A prevalência de L15 nos utilizadores com IMC $<25$ foi de $3,06 \%(\mathrm{H}=2,81 \%, \mathrm{M}=3,23 \%)$, nos com IMC $=25-29,9$ foi de $3,51 \%(\mathrm{H}=2,91 \%, \mathrm{M}=4,03 \%)$ e naqueles com IMC $\geq 30$ foi de $4,87 \%$ ( $\mathrm{H}=3,67 \%, M=5,58 \%)$ (Quadro II).

Relativamente a L15, a diferença entre a prevalência dos utilizadores com IMC $<25$ e com IMC=25-29,9 é es- 


\begin{tabular}{|c|c|c|c|c|c|c|c|c|c|c|c|c|}
\hline & \multicolumn{3}{|c|}{$I M C<25$} & \multicolumn{3}{|c|}{$I M C=25-29,9$} & \multicolumn{3}{|c|}{$I M C \geq 30$} & \multicolumn{3}{|c|}{ TOTAL } \\
\hline & $\mathrm{H}$ & $M$ & Total & $\mathrm{H}$ & $M$ & Total & $\mathrm{H}$ & $M$ & Total & $\mathrm{H}$ & $M$ & Total \\
\hline $21-30$ anos & 65.366 & 112131 & 177497 & 2385 & 3581 & 5966 & 2054 & 4548 & 6602 & 69805 & 120260 & 190065 \\
\hline $31-40$ anos & 86149 & 153991 & 240140 & 5920 & 7089 & 13009 & 5326 & 9674 & 15000 & 97395 & 170754 & 268149 \\
\hline $41-50$ anos & 93843 & 146670 & 240513 & 8425 & 9825 & 18250 & 9227 & 13829 & 23056 & 111495 & 170324 & 281819 \\
\hline $51-60$ anos & 97411 & 137269 & 234680 & 11296 & 12971 & 24267 & 12136 & 19489 & 31625 & 120843 & 169729 & 290572 \\
\hline $61-70$ anos & 108008 & 138020 & 246028 & 15244 & 16192 & 31436 & 14857 & 23810 & 38667 & 138109 & 178022 & 316131 \\
\hline $71-80$ anos & 86215 & 115218 & 201433 & 12384 & 13370 & 25754 & 9778 & 17565 & 27343 & 108377 & 146153 & 254530 \\
\hline \multirow[t]{2}{*}{$>80$ anos } & 41690 & 79730 & 121420 & 4261 & 6435 & 10696 & 2637 & 6451 & 9088 & 48588 & 92616 & 141204 \\
\hline & 578682 & 883029 & 1461711 & 59915 & 69463 & 129378 & 56015 & 95366 & $\mid 151381$ & | 694612 & 1047858 & 1742470 \\
\hline
\end{tabular}

\begin{tabular}{|c|c|c|c|c|c|c|c|c|c|c|c|c|c|}
\hline \multirow{2}{*}{\multicolumn{2}{|c|}{ Utilizadores }} & \multicolumn{3}{|c|}{ IMC $<25$} & \multicolumn{3}{|c|}{$I M C=25-29,9$} & \multicolumn{3}{|c|}{$I M C \geq 30$} & \multicolumn{3}{|c|}{ TOTAL } \\
\hline & & $\mathrm{H}$ & $M$ & Total & $\mathrm{H}$ & $M$ & Total & $\mathrm{H}$ & $M$ & Total & $\mathrm{H}$ & $M$ & Total \\
\hline \multirow[t]{2}{*}{ L13 } & $\mathbf{N}$ & 6018 & 15529 & 21547 & 608 & 1433 & 2041 & 602 & 2128 & 2730 & 7228 & 19090 & 26318 \\
\hline & $\%$ & 1,04 & 1,76 & 1,47 & 1,01 & 2,06 & 1,58 & 1,07 & 2,23 & 1,80 & 1,04 & 1,82 & 1,51 \\
\hline \multirow[t]{2}{*}{ L15 } & $\mathbf{N}$ & 16234 & 28547 & 44781 & 1743 & 2802 & 4545 & 2053 & 5324 & 7377 & 20030 & 36673 & 56703 \\
\hline & $\%$ & 2,81 & 3,23 & 3,06 & 2,91 & 4,03 & 3,51 & 3,67 & 5,58 & 4,87 & 2,88 & 3,50 & 3,25 \\
\hline \multirow[t]{2}{*}{ L89 } & $\mathbf{N}$ & 10192 & 20847 & 31039 & 2126 & 3703 & 5829 & 2241 & 5746 & 7987 & 14559 & 30296 & 44855 \\
\hline & $\%$ & 1,76 & 2,36 & 2,12 & 3,55 & 5,33 & 4,51 & 4,00 & 6,03 & 5,28 & 2,10 & 2,89 & 2,57 \\
\hline \multirow[t]{2}{*}{ L90 } & $\mathbf{N}$ & 18323 & 44006 & 62329 & 3782 & 7670 & 11452 & 4871 & 16477 & 21348 & 26976 & 68153 & 95129 \\
\hline & $\%$ & 3,17 & 4,98 & 4,26 & 6,31 & 11,04 & 8,85 & 8,70 & 17,28 & 14,10 & 3,88 & 6,50 & 5,46 \\
\hline
\end{tabular}

tatisticamente significativa (OR=1,15; IC95\%: 1,12-1,19; $p<0,0001)$, assim como também o é a dos com IMC $<25$ e com IMC $\geq 30$ (OR=1,62; IC95\%: 1,58-1,66; $p<0,0001)$. No género masculino, a diferença entre a prevalência dos utilizadores com IMC $<25$ e com IMC=25-29,9 não é estatisticamente significativa (Quadro III).

\section{Osteoartrose da anca (L89)}

Apresentaram osteoartrose da anca 2,57\% ( $n=44.855)$ dos utilizadores $-2,10 \%$ ( $n=14.559)$ do género masculino e $2,89 \%$ ( $n=30.296$ ) do género feminino. A prevalência de L89 nos utilizadores com IMC $<25$ foi de 2,12\%
$(\mathrm{H}=1,76 \%, \mathrm{M}=2,36 \%)$, nos com $\mathrm{IMC}=25-29,9$ foi $\mathrm{de}$ $4,51 \%(\mathrm{H}=3,55 \%, \mathrm{M}=5,33 \%)$ e naqueles com $\mathrm{IMC} \geq 30$ foi de 5,28\% (H=4,00\%, M=6,03\%) (Quadro II).

Relativamente a L89, a diferença entre a prevalência dos utilizadores com IMC $<25$ e com IMC=25-29,9 é estatisticamente significativa (OR=2,17; IC95\%: 2,11-2,24; $p<0,0001)$, assim como o é a dos com IMC $<25$ e com IMC $\geq 30$ (OR=2,57; IC95\%: 2,50-2,63; $p<0,0001$ ) (Quadro III).

\section{Osteoartrose do joelho (L90)}

Apresentaram osteoartrose do joelho 5,46\% 


\begin{tabular}{|c|c|c|c|c|c|c|c|}
\hline \multirow{3}{*}{$\begin{array}{l}\text { QUAD } \\
\text { p-valo } \\
\text { (L13 - } \\
\text { (L15 - } \\
\text { IMC e } \\
\text { ICPC-2 }\end{array}$} & $\begin{array}{l}\text { III. O } \\
\text { ) para } \\
\text { toma } \\
\text { toma } \\
\text { géner }\end{array}$ & eixa & $\begin{array}{l}\text { com inte } \\
\text { s do ICPC } \\
\text { anca, L8 } \\
\text { joelho, L }\end{array}$ & $\begin{array}{l}\text { los de co } \\
\text { relaciona } \\
\text { osteoart } \\
\text { - osteoa }\end{array}$ & $\begin{array}{l}\text { Tça } \\
\text { com } \\
\text { da a } \\
\text { se dc }\end{array}$ & $\begin{array}{l}5 \% \text { (IC9 } \\
\text { bblemas } \\
\text { a) e do jo } \\
\text { elho) em }\end{array}$ & $\begin{array}{l}\text { e } \\
\text { inca } \\
0 \\
\text { ção do }\end{array}$ \\
\hline & & \multicolumn{3}{|c|}{$\mathrm{IMC}=25-29,9$} & \multicolumn{3}{|c|}{$I M C \geq 30$} \\
\hline & & OR & IC (95\%) & $p$ & OR & IC (95\%) & $P$ \\
\hline & Total & 1,07 & $1,02-1,12$ & $p<0,0001$ & 1,23 & $1,18-1,28$ & $p<0,0001$ \\
\hline \multirow[t]{3}{*}{ L13 } & $\mathrm{H}$ & 0,98 & $0,90-1,06$ & $p=0,5626$ & 1,03 & $0,95-1,12$ & $p=0,5981$ \\
\hline & $M$ & 1,18 & $1,11-1,24$ & $p<0,0001$ & 1,27 & $1,22-1,33$ & $p<0,0001$ \\
\hline & Total & 1,15 & $1,12-1,19$ & $p<0,0001$ & 1,62 & $1,58-1,66$ & $p<0,0001$ \\
\hline \multirow[t]{3}{*}{ L15 } & $\mathrm{H}$ & 1,04 & 0,99-1,09 & $p=0,1437$ & 1,32 & $1,26-1,38$ & $p<0,0001$ \\
\hline & $M$ & 1,26 & $1,21-1,31$ & $p<0,0001$ & 1,77 & $1,72-1,82$ & $p<0,0001$ \\
\hline & Total & 2,17 & $2,11-2,24$ & $p<0,0001$ & 2,57 & $2,50-2,63$ & $p<0,0001$ \\
\hline \multirow[t]{3}{*}{ L89 } & $\mathrm{H}$ & 2,05 & $1,96-2,15$ & $p<0,0001$ & 2,32 & $2,22-2,44$ & $p<0,0001$ \\
\hline & $M$ & 2,33 & $2,25-2,41$ & $p<0,0001$ & 2,65 & $2,57-2,73$ & $p<0,0001$ \\
\hline & Total & 2,18 & $2,14-2,23$ & $p<0,0001$ & 3,69 & $3,63-3,75$ & $p<0,0001$ \\
\hline \multirow[t]{2}{*}{ L90 } & $\mathrm{H}$ & 2,06 & $1,99-2,14$ & $p<0,0001$ & 2,91 & $2,82-3,01$ & $p<0,0001$ \\
\hline & $M$ & 2,37 & $2,31-2,43$ & $p<0,0001$ & 3,98 & $3,91-4,06$ & $p<0,0001$ \\
\hline
\end{tabular}

( $n=95.129$ ) dos utilizadores, dos quais 3,88\% ( $n=26.976$ ) do género masculino e $6,50 \%$ ( $n=68.153)$ do género feminino. A prevalência de L90 nos utilizadores com IMC $<25$ foi de $4,26 \%$ ( $\mathrm{H}=3,17 \%, \mathrm{M}=4,98 \%)$, nos com IMC $=25-29,9$ foi de $8,85 \%$ ( $\mathrm{H}=6,31 \%, \mathrm{M}=11,04 \%)$ e naqueles com IMC $\geq 30$ foi de $14,10 \%$ ( $\mathrm{H}=8,70 \%$, $\mathrm{M}=17,28 \%$ ) (Quadro II).

Relativamente a L90, a diferença entre a prevalência dos utilizadores com IMC $<25$ e com IMC=25-29, 9 é estatisticamente significativa (OR=2,18; IC95\%: 2,14-2,23; $p<0,0001)$, assim como também o é a dos com IMC $<25$ e com IMC $\geq 30$ (OR=3,69; IC95\%: 3,63-3,75; $p<0,0001)$ (Quadro III).

\section{DISCUSSÃO}

A prevalência de sintoma/queixa da anca nos utilizadores com excesso de peso, assim como naqueles com obesidade, foi maior do que nos utilizadores com $\mathrm{IMC}<25$, havendo uma associação positiva entre o excesso de peso e sintoma/queixa da anca e a obesidade e sintoma/queixa da anca. Existem poucos estudos relacionando o excesso de peso e a obesidade com sin- toma/queixa da anca. Contudo, o estudo de Adamson e colaboradores ${ }^{6}$ refere uma relação positiva entre a obesidade e a dor da anca e Ackerman e Osborne ${ }^{7}$ referem no seu que os indivíduos com obesidade e coxartrose reportaram mais dor, maior rigidez, pior função, um resultado no HRQoL (Health Related Quality of Life) mais baixo e uma maior gravidade da doença.

A prevalência de sintoma/queixa do joelho nos utilizadores com excesso de peso e naqueles com obesidade foi maior do que a prevalência nos utilizadores com IMC $<25,0$, havendo uma associação positiva entre excesso de peso e sintoma/queixa do joelho e entre obesidade e sintoma/queixa do joelho. O estudo pioneiro sobre a relação entre o IMC e a sintomatologia do joelho é o de Felson e colaboradores, ${ }^{8}$ no qual foi demonstrado que uma perda ponderal reduz o risco de gonartrose sintomática em mulheres. Webb e colaboradores ${ }^{9}$ reportam no seu estudo que os participantes obesos tiveram quase quatro vezes mais queixas de dores no joelho acompanhadas de incapacidade do que os participantes com IMC normal, ajustados pela idade e género (AOR: $3,63,2,48-5,30)$, e que o excesso de peso e a obesidade foram responsáveis por um quinto dos casos de gonalgia, um terço dos casos de gonalgia com incapacidade e um terço dos casos graves de gonalgia com incapacidade. Ackerman e Osborne ${ }^{7}$ reportam no seu estudo que os participantes obesos com gonartrose e dor referiram maior rigidez, pior função e uma maior gravidade da doença.

A prevalência da coxartrose nos utilizadores com excesso de peso e naqueles com obesidade foi maior do que a dos com IMC $<25$, havendo uma associação positiva entre excesso de peso e coxartrose e entre obesidade e coxartrose. Jiang e colaboradores, ${ }^{10}$ numa revisão sistemática e meta-análise sobre a relação entre IMC e coxartrose, confirmam uma associação moderada entre obesidade e coxartrose e reportam que, para 
um aumento de cinco unidades no IMC, o risco de coxartrose aumenta em 11\% (RR=1,11; IC95\%: 1,07-1,16); não houve diferença significativa na magnitude da associação para o género. Lohmander e colaboradores, ${ }^{11}$ num estudo de coorte de onze anos para determinar a relação entre diferentes medidas de IMC e a incidência de artrose grave da anca, definida como artroplastia devido a osteoartrose, reportaram que o IMC estava significativamente associado $[R R=2,6(2,0-3,4)]$ com a incidência de artroplastia.

No estudo, a prevalência da gonartrose nos utilizadores com excesso de peso e com obesidade foi bastante maior do que a dos utilizadores com IMC $<25$, havendo uma associação positiva entre o excesso de peso e a gonartrose, assim como entre obesidade e gonartrose. Estes resultados estão de acordo com os referidos em diversos estudos, em que a associação do excesso de peso e da obesidade com a gonartrose foi consistentemente demonstrada. Assim, Coggon e colaboradores $^{12}$ relataram que indivíduos com IMC $>30$ foram 6,8 vezes mais propensos a desenvolver gonartrose do que os controlos de peso normal; e March e Bagga $^{13}$ demonstraram que o risco de gonartrose aumenta $36 \%$ por cada duas unidades de IMC $(5 \mathrm{Kg})$ de ganho ponderal. Numa revisão sistemática e metaanálise, Blagojevic e colaboradores ${ }^{14}$ demonstraram que em todos os 36 estudos incluídos o excesso de peso e a obesidade foram fatores de risco para a gonartrose (RR=2,63; IC95\%: 2,28-3,05). Jiang e colaboradores, ${ }^{15}$ numa outra revisão sistemática e meta-análise sobre a relação entre IMC e gonartrose, confirmam uma associação entre obesidade e gonartrose e reportam que, para um aumento de cinco unidades no IMC, o risco de gonartrose aumenta em 35\% $(\mathrm{RR}=1,35$; IC95\%: 1,21-1,51); esta associação é significativamente mais forte nas mulheres (RR=1,38; IC95\%: 1,23-1,54; $p=0,04)$ do que nos homens (RR=1,22; IC95\%: 1,19$1,25 ; p=0,04)$.

Podemos, pois, concluir que os resultados obtidos neste estudo vieram confirmar para a população portuguesa participante os resultados de estudos internacionais, isto é, de que existe uma associação positiva entre a dor músculo-esquelética e a osteoartrose da anca e do joelho nos indivíduos com excesso de peso e com obesidade. Estes resultados, obtidos a partir de uma percentagem alargada da população portuguesa
$(16,7 \%)$, poderão ser mais uma chamada de atenção e um incentivo para que os médicos de MGF e todos os outros profissionais de saúde tenham uma especial atenção à prevalência do excesso ponderal nos indivíduos a seu cargo, implementando as convenientes e necessárias medidas de prevenção e de tratamento para um efetivo controlo deste importante problema com impacto na saúde e na qualidade de vida das populações.

\section{REFERÊNCIAS BIBLIOGRÁFICAS}

1. World Health Organization. Obesity and overweight [Internet]. Geneva: WHO [updated 2016 Jun; cited 2016 Nov 17]. Available from: http://www.who.int/mediacentre/factsheets/fs311/en/

2. Dawson L, Wluka AE, Wang Y, Cicuttini FM. Obesity, arthritis, and gout. In: Bray GA, Bouchard C, editors. Handbook of obesity. Vol. 1: epidemiology, etiology, and physiopathology. Boca Raton: CRC Press; 2014. p. 625-33. ISBN 9781842145562

3. King LK, March L, Anandacoomarasamy A. Obesity \& osteoarthritis. Indian J Med Res. 2013;138(2):185-93.

4. Bonakdar RA. Targeting systemic inflammation in patients with obesity-related pain: obesity- related pain: time for a new approach that targets systemic inflammation. J Fam Pract. 2013;62(9 Suppl CHPP):S229 .

5. Administração Central do Sistema de Saúde. Classificação internacional de cuidados de saúde primários - Segunda edição [Internet]. Lisboa: ACSS; 2011. Available from: http://www.acss.min-saude.pt/wpcontent/uploads/2016/07/1_ICPC_2_4_4_VF.pdf

6. Adamson J, Ebrahim S, Dieppe P, Hunt K. Prevalence and risk factors for joint pain among men and women in the West of Scotland Twenty-07 study. Ann Rheum Dis. 2006;65(4):520-4.

7. Ackerman IN, Osborne RH. Obesity and increased burden of hip and knee joint disease in Australia: results from a national survey. BMC Musculoskelet Disord. 2012;13:254.

8. Felson DT, Zhang Y, Anthony JM, Naimark A, Anderson JJ. Weight loss reduces the risk for symptomatic knee osteoarthritis in women: the Framingham Study. Ann Intern Med. 1992;116(7):535-9.

9. Webb R, Brammah T, Lunt M, Urwin M, Allison T, Symmons D. Opportunities for prevention of 'clinically significant' knee pain: results from a population-based cross sectional survey. J Public Health (Oxf). 2004;26(3):277-84.

10. Jiang L, Rong J,Wang Y, Hu F, Bao C, Li X, et al. The relationship between body mass index and hip osteoarthritis: a systematic review and metaanalysis. Joint Bone Spine. 2011;78(2):150-5.

11. Lohmander LS, Gerhardsson de Verdier M, Rollof J, Nilsson PM, Engström $\mathrm{G}$. Incidence of severe knee and hip osteoarthritis in relation to different measures of body mass: a population-based prospective cohort study. Ann Rheum Dis. 2009;68(4):490-6.

12. Coggon D, Reading I, Croft P, McLaren M, Barrett D, Cooper C. Knee osteoarthritis and obesity. Int J Obes Relat Metab Disord. 2001;25(5):6227.

13. March LM, Bagga H. Epidemiology of osteoarthritis in Australia. Med J 
Aust. 2004;180(5 Suppl):S6-10.

14. Blagojevic M, Jinks C, Jeffery A, Jordan KP. Risk factors for onset of osteoarthritis of the knee in older adults: a systematic review and metaanalysis. Osteoarthritis Cartilage. 2010;18(1):24-33.

15. Jiang L, Tian W, Wang Y, Rong J, Bao C, Liu Y, et al. Body mass index and susceptibility to knee osteoarthritis: a systematic review and metaanalysis. Joint Bone Spine. 2012;79(3):291-7.

\section{CONFLITO DE INTERESSES}

Os autores declaram não ter conflitos de interesse.

\section{COMISSÃO DE ÉTICA}

Estudo realizado após parecer favorável da Comissão de Ética para a Saúde da ARSLVT.

\section{ENDEREÇO PARA CORRESPONDÊNCIA}

Andreia Baptista Marques

E-mail: anbmarques@outlook.pt

Recebido em 20-03-2016

Aceite para publicação em 25-04-2017

\section{ABSTRACT}

\section{THE ASSOCIATION BETWEEN OVERWEIGHT, OBESITY, MUSCULOSKELETAL PAIN AND OSTEOARTHRITIS IN PRIMARY HEALTH CARE: CROSS-SECTIONAL STUDY}

Objectives: To evaluate the association of overweight, obesity, musculoskeletal pain and osteoarthritis in primary care.

Design: Cross-sectional study.

Setting: Health units in the Group of Health Centres of the Lisboa e Vale do Tejo Regional Health Administration

Participants: Patients aged 21 years or older who consulted with a family doctor in 2014.

Methods: Data were obtained from the centralized computer records of the regional health administration (Sistema de Informação da Administração Regional de Saúde). ICPC-2 codes L13, L15, L89, L90, T82 and T83, were examined by gender and age group. Descriptive statistics and chi-square tests were applied to evaluate differences in prevalence between the variables, with a significance level of 0.05 .

Results: The prevalences of overweight and obesity in the $1,742.470$ patients in this population were $7.42 \%$ and $8.69 \%$. The prevalence of hip symptoms or complaints was $1.51 \%$, knee symptoms or complaint $3.25 \%$, osteoarthritis of the hip $2.57 \%$, and osteoarthritis of the knee $5.46 \%$. There was a statistically significant association between overweight and hip symptoms or complaints (OR=1.07; IC95\%: 1.02-1.12, $p<0.0001)$, knee symptoms or complaints (OR=1.15; IC95\%: 1.12-1.19, $p<0.0001)$, osteoarthritis of the hip (OR=2.17; IC95\%: 2.11-2.24, $p<0.0001)$ and osteoarthritis of the knee (OR=2.18; IC95\%: 2.14-2.23, $p<0.0001)$, and between obesity and hip symptoms or complaints ( $O R=1.23 ; \mathrm{IC95} \%: 1.18-1.28, p<0.0001)$, knee symptoms or complaints (OR=1.62; IC95\%: 1.58-1.66, $p<0.0001)$, osteoarthritis of the hip (OR=2.57; IC95\%: 2.50-2.63, $p<0.0001)$, and osteoarthritis of the knee (OR=3.69; IC95\%: 3.63-3.75, $p<0.0001)$.

Conclusions: The positive association between overweight and obesity, and hip symptoms or complaints, knee symptoms or complaints, osteoarthritis of the hip, and osteoarthritis of the knee in this population are consistent with results from international studies.

Keywords: Obesity; Musculoskeletal pain; Osteoarthritis; Association; Primary health care. 